Check for updates

Cite this: RSC Adv., 2017, 7, 24382

Received 25th January 2017

Accepted 21st April 2017

DOI: 10.1039/c7ra01107e

rsc.li/rsc-advances

\title{
Concentration-related microwave heating processes: electromagnetic interference of Maillard reaction substrates (glucose and lysine)
}

\author{
Nana Zhang, ${ }^{a}$ Daming Fan, (D) *a Meng Chen, ${ }^{a}$ Yanfang Chen, ${ }^{a}$ Jianlian Huang, ${ }^{b}$

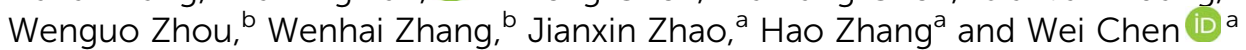

\begin{abstract}
Glucose and lysine are important substrates involved in the Maillard reaction. The lack of studies on their electromagnetic properties has made it impossible to understand the influence of electromagnetic properties on the microwave heating process, and has hindered the application of the Maillard reaction in the microwave field. In this study, the electromagnetic properties of glucose, lysine solution and their $1: 1$ mixture were determined at frequencies of $1.7-2.6 \mathrm{GHz}$. The results show that the dielectric loss factors gradually increased. Reflection loss gradually decreased with concentration in the order of glucose $>$ mixture $>$ lysine. The heating rates gradually increased at low concentrations $(\leq 5 \%)$ and slowly changed at high concentrations $(\geq 10 \%)$. These results indicate that the effect of electromagnetic properties on the heating rate is related to concentration. The heating rates depended on the dielectric properties at low concentrations $(\leq 5 \%)$, whereas microwave-absorption properties dominated at high concentrations ( $\geq 10 \%)$
\end{abstract}

\section{Introduction}

Complex permittivity is a basic electromagnetic property that reflects the microscopic nature of a material but it cannot directly reflect the response of the material under a microwave field due to the impedance matching problem. The microwaveabsorption properties of a material are the comprehensive embodiment of its dielectric properties, magnetic properties and macroscopic morphology and reflect the response of the material under a microwave field. ${ }^{1}$ Reflection loss (RL) is an important parameter in the evaluation of a material's absorption performance, and can be tested by the arch method. ${ }^{2-4}$ In a Maillard reaction system, the RL of a substrate characterizes its response to an incident electromagnetic wave in terms of absorption behavior and release and loss of the electromagnetic wave, indirectly affecting the degree of Maillard reaction under a microwave field.

Glucose and lysine are important substrates involved in the Maillard reaction. Reaction temperature and time are the most important factors influencing the degree of Maillard reaction. ${ }^{5}$ The electromagnetic properties of the substrates indirectly regulate the heating process, thus affecting the progress of the Maillard reaction in a microwave field. More importantly, the two amino structures of lysine allow it to have a strong

${ }^{a}$ State Key Laboratory of Food Science and Technology, School of Food Science and Technology, Jiangnan University, Wuxi 214122, China. E-mail: fandm@jiangnan. edu.cn; Fax: +860510 85912155; Tel: +86051085884620

${ }^{b}$ Fujian Anjoyfood Share Co. Ltd., Xiamen 361022, China response under a microwave field, which makes it possible to produce an unpredictable response after mixing with glucose. However, most studies have focused on the dielectric properties of these substances. The lack of studies on their microwave-absorption properties has made it impossible to understand the influence of electromagnetic properties on the heating process, and has indirectly hindered the application of the Maillard reaction under a microwave field. Many studies have reported experimental methods. ${ }^{6,7}$ and have theorized about liquid dielectric properties. ${ }^{8,9}$ The effects of concentration, temperature, and frequency on dielectric properties have been studied, and relevant theoretical or empirical models have been established. Liao et al. (2003) have found that the dielectric properties of $\alpha$-D-glucose solution are related to temperature and concentration. ${ }^{10}$ In addition, from a study of the dielectric properties of lysine solutions, it can be seen that in a certain concentration range, the dielectric constant decreases with increasing concentration and the dielectric loss factor gradually increases. ${ }^{11}$

However, no research has focused on the microwaveabsorption properties of glucose, lysine and their mixtures, which limits the application of the Maillard reaction under a microwave field. In this paper, we report our investigation of the relationship between concentration and microwaveabsorption properties based on dielectric properties, and further clarify the influence of electromagnetic properties on heating, with the aim of optimizing the microwave heating method to promote Maillard reactions under a microwave field. 


\section{Materials and methods}

Materials

D-glucose and L-lysine (Sinopharm Chemical Reagent Company, Shanghai, China) were used without purification.

\section{Glucose, lysine and their $1: 1$ mixed solutions}

Solutions were prepared by adding glucose or lysine to suitable bottles, which were filled up with distilled water to the specific weight. Three different solutions at 1\%, 3\%, 5\%, 10\% and 15\% $(\mathrm{w} / \mathrm{w})$ concentrations were prepared and magnetically stirred at room temperature until completely dissolved. The $1: 1$ glucose/ lysine mixture reflects the common concentration of the Maillard reaction.

\section{Dielectric properties measurements}

The dielectric properties of the liquid systems were measured using a vector network analyzer (E5071C, Agilent, Santa Clara, CA, USA) with an open-ended coaxial line, connected to

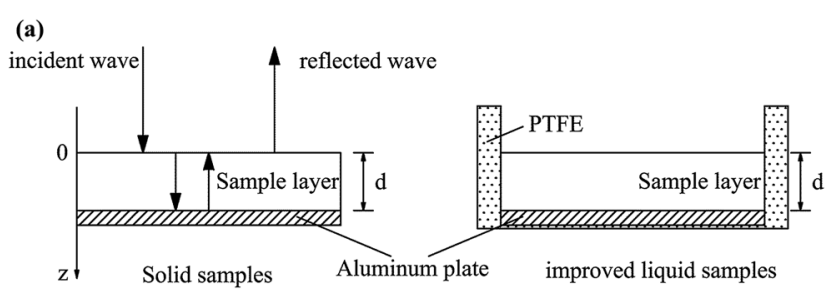

(b)

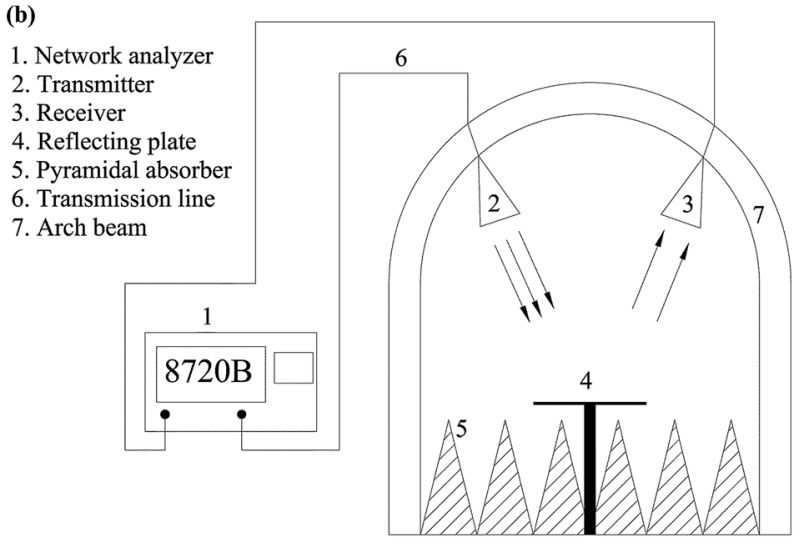

Fig. 1 The improved specialized container for liquid samples (a) and a schematic diagram of the arch method testing system (b). a dielectric probe (85070E, Agilent, Santa Clara, CA, USA). ${ }^{\mathbf{1 2 , 1 3}}$ The probe was calibrated with air, a short-circuit and water, respectively. Each sample was measured in triplicate.

\section{Electrical conductivity measurements}

Room-temperature electrical conductivity testing of the samples was carried out using a conductivity meter (LA-EC20, HACH, USA). The probe was calibrated with a calibration solution. Each sample was measured in triplicate.

\section{RL measurements}

The RL versus frequency for liquid materials was improved based on the arch method by adding polytetrafluoroethylene (PTFE) as a wall material on an aluminum plate, to make a specialized container (Fig. 1a). The testing system of the improved arch method included a vector network analyzer (HP 8720B, Hewlett Packard Co., Palo Alto, CA, USA) and standard horn antennas in an anechoic chamber as a function of frequency from 1.7 to $2.6 \mathrm{GHz}$ (Fig. 1b). ${ }^{\mathbf{1 4}}$

\section{Heating curve measurements}

Each solution sample weighing $1.0 \pm 0.005 \mathrm{~g}$ was uniformly distributed into lidless quartz test tubes (inner diameter $=12$ $\mathrm{mm})$. The samples were heated with a microwave at $150 \mathrm{~W} \mathrm{~g}^{-1}$ for $16 \mathrm{~s}$ (as determined by earlier experiments) using a MultiSYNTH microwave synthesizer (Milestone, Sorisole, Italy) set to a frequency of $2450 \mathrm{MHz} .{ }^{15}$ An integrated infrared temperature probe was used to detect the temperature of the samples. Each experiment was conducted three times.

\section{Results and discussion}

\section{Effect of concentration on dielectric properties}

At room temperature, the dielectric constants for glucose, lysine and the $1: 1$ mixed solution generally decreased as concentration increased, as shown in Fig. 2a. This observation is similar to those documented in previous reports. ${ }^{10,11,16}$ For the three different solutions, the $\varepsilon^{\prime}$ showed little difference at the same concentration, and the variation of $\varepsilon^{\prime}$ with concentration was linear and significant $(P<0.0001)$ with coefficients of variation of $2.5 \%, 4.1 \%$ and $4.0 \%$, respectively (see Fig. $2 \mathrm{a}$ ). The linearity of the $\varepsilon^{\prime}$-concentration relationship indicates that as far as the
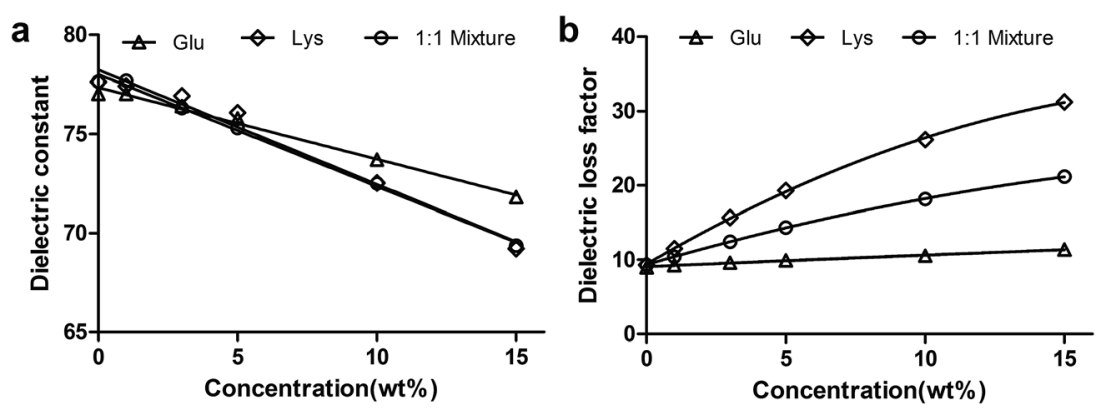

Fig. 2 Influence of concentration on the dielectric constant (a) and dielectric loss factor (b) of the three different solutions. 


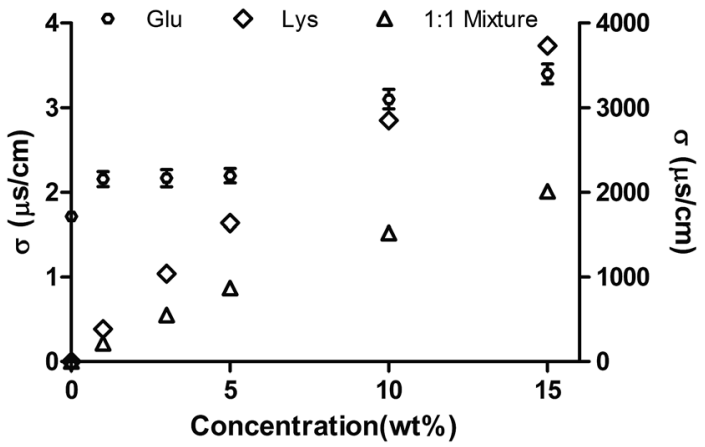

Fig. 3 Influence of concentration on the electrical conductivity of the three different solutions (glucose solution, left $y$-axis; lysine solution and $1: 1$ mixture, right $y$-axis).

dielectric constant is concerned there is no interaction between the two components.

The influence of concentration on loss factor at room temperature is shown in Fig. 2b. For the glucose solution, the loss factor increased linearly with concentration across the whole concentration range studied. This observation is similar to previous reports. ${ }^{10,11}$ Similar to the results for the dielectric constant, the linear relationship between loss factor and concentration suggests that the loss factor of this two-component system is contributed directly from each individual component. No interaction exists between water and glucose molecules. However, the loss factor of lysine solution and the 1:1 mixture showed a quadratic increase with concentration in the order of lysine $>$ mixture $>$ glucose. The increase may have resulted from an increase in the number of free molecules.

\section{Effect of concentration on electrical conductivity}

At room temperature, the electrical conductivity $(\sigma)$ of the three different solutions generally increased with the increasing of concentration, as shown in Fig. 3. Across the whole concentration

Table 1 Heating rates of the three different solutions

\begin{tabular}{lcccccc}
\hline & Water & $1 \%$ & $3 \%$ & $5 \%$ & $10 \%$ & $15 \%$ \\
\hline Lysine & 3.81 & 3.94 & 4.46 & 4.74 & 4.94 & 5.28 \\
Glucose & 3.81 & 3.84 & 4.06 & 4.49 & 5.67 & 5.96 \\
$1: 1$ mixture & 3.81 & 3.92 & 4.36 & 4.67 & 5.50 & 5.76
\end{tabular}

a

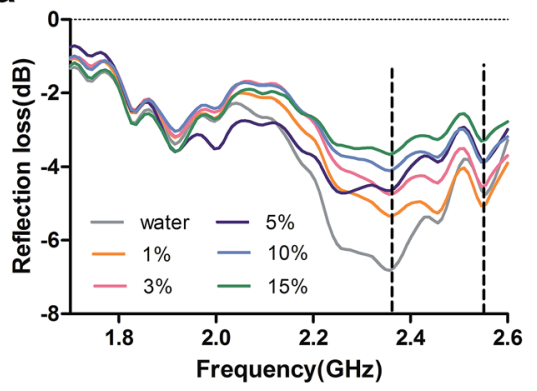

c

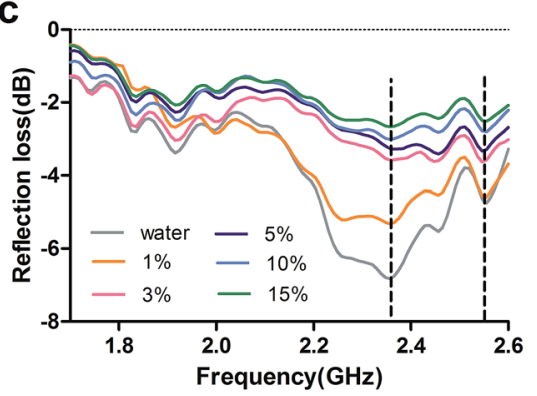

e

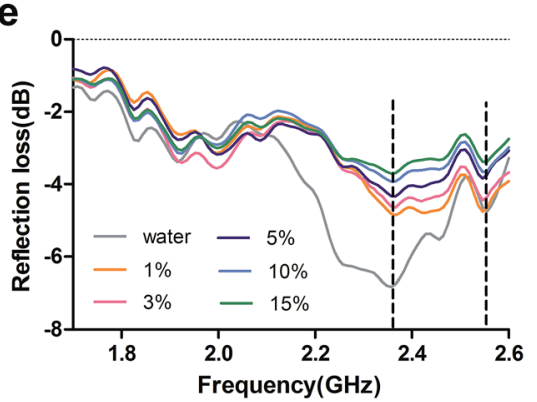

b

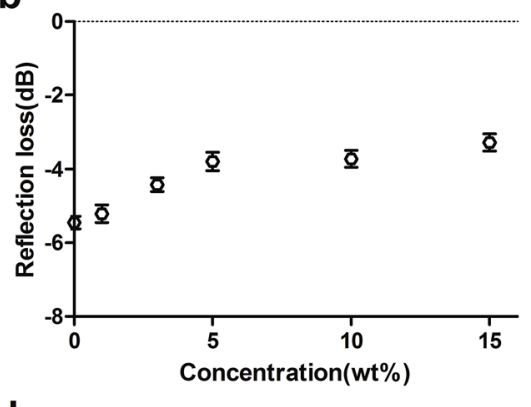

d

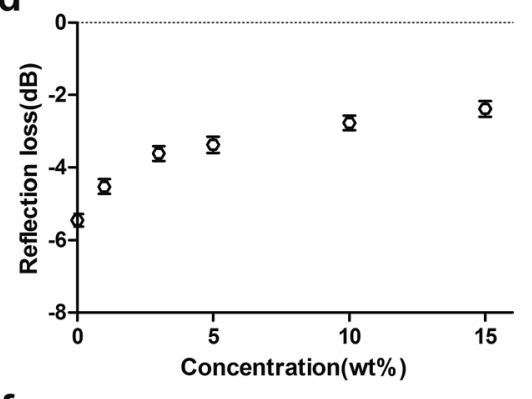

f

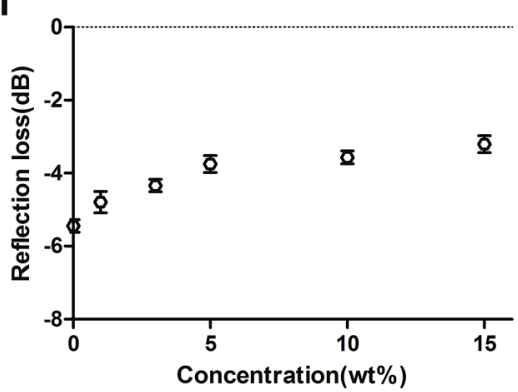

Fig. 4 Reflection loss of glucose (a), lysine solutions (c) and $1: 1$ mixtures (e) at frequencies of 1.7-2.6 GHz and the RL curve at 2.45 GHz for glucose (b), lysine solutions (d) and $1: 1$ mixtures ( $f$ ) of different concentrations at room temperature. 

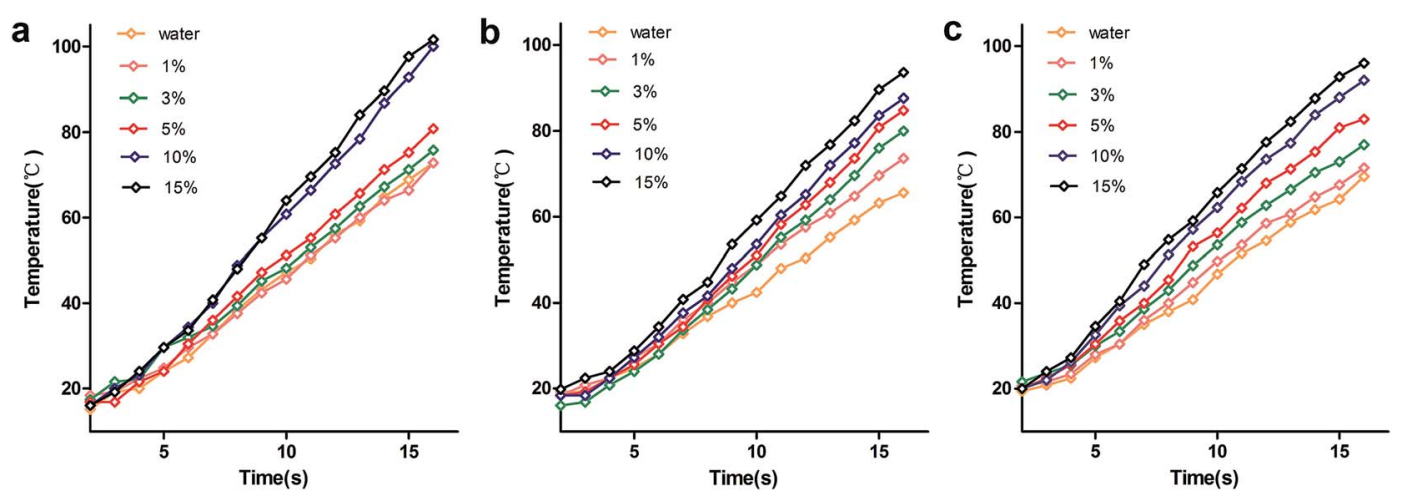

Fig. 5 Heating curves of glucose (a), lysine solutions (b) and 1: 1 mixtures (c) with different concentrations.

range studied, the electrical conductivity of the solutions was in the order of lysine $>$ mixture $>$ glucose, which can be attributed to the number of free amino groups. From the Debye theory of dielectrics, it is known that the permittivity of a dielectric material increases with increasing electrical conductivity. ${ }^{17,18}$ The relationship between the imaginary part of permittivity and electrical conductivity was very consistent with the theory in this study. The imaginary part of permittivity represents the dielectric loss ability of materials. Therefore, it can be explained by the increase in dielectric loss with higher electrical conductivity, which is similar to previous report. ${ }^{19}$

\section{Effect of concentration on reflection loss}

Reflection loss is an important parameter with which to characterize the absorption properties of materials. ${ }^{20}$ We investigated the RL curves of $3 \mathrm{~mm}$-thick glucose (Fig. 4a), lysine solutions (Fig. 4c) and 1:1 mixtures (Fig. 4e) at frequencies of 1.7-2.6 GHz. For glucose solutions and $1: 1$ mixtures, water and all solutions with different concentrations showed the same absorption peak at $2.37 \mathrm{GHz}$, suggesting that water molecules dominated the electromagnetic properties of the solutions. However, the absorption peak of the lysine solutions was shifted to the right (from $2.37 \mathrm{GHz}$ to $2.55 \mathrm{GHz}$ ) as the concentration increased, which suggests that lysine molecules gradually dominate the electromagnetic properties of the mixture, which is similar to previous report. ${ }^{\mathbf{1 4}}$

At a frequency of 2.4-2.5 GHz, the RL of the three different solutions generally decreased with increasing concentration, and RL at 2.45 GHz also meet the rule as shown in Fig. $4 \mathrm{~b}$, $\mathrm{d}$ and e. The RL of the solutions was in the order of glucose $>$ mixture $>$ lysine, and showed only a small change at concentrations above $5 \%$, which may be due to poor impedance matching at the free space/materials interface. , $121^{21}$

\section{Effect of concentration on heating curve}

To understand the effects of dielectric and microwaveabsorption properties on the heating curve, heating curves of glucose (Fig. 5a), lysine solutions (Fig. 5b) and 1:1 mixtures (Fig. 5c) with different concentrations were measured. Across the whole concentration range studied, the heating rate was in the order of lysine $>$ mixture $>$ glucose at low concentrations $(\leq 5 \%)$, and glucose $>$ mixture $>$ lysine at high concentrations $(\geq 10 \%)$, as shown in Table 1 . The results indicate that the heating rate is related to concentration. Combined with the dielectric and microwave-absorption properties, the dielectric loss factors were in the order of lysine $>$ mixture $>$ glucose at low concentrations $(\leq 5 \%)$, and RL was in the order of glucose $>$ mixture $>$ lysine at high concentrations $(\geq 10 \%)$. These results indicate that heating depends on dielectric properties at low concentrations $(\leq 5 \%)$ but it is dominated by microwaveabsorption properties at high concentrations $(\geq 10 \%)$.

\section{Conclusions}

The dielectric properties of D-glucose, L-lysine solutions and their mixtures were shown to be dependent on concentration. The dielectric loss factors gradually increased and show the order of lysine $>$ mixture $>$ glucose. The results can be explained by the increase in dielectric loss factor with higher electrical conductivity. RL gradually decreased with concentration in the order of glucose $>$ mixture $>$ lysine at frequencies of $2.4-2.5 \mathrm{GHz}$.

The heating rates showed that the effect of electromagnetic properties is related to concentration. It was gradually increased at low concentrations $(\leq 5 \%)$ and depended on the dielectric properties, while slowly changed at high concentrations $(\geq 10 \%$ ), in which the effect was dominated by the microwaveabsorption properties. Further studies could focus on the effect of different microwave heating conditions on electromagnetic properties.

\section{Acknowledgements}

This work was supported by National Natural Science Foundation of China (Grant No. 31571879, 31301504), the "FiveTwelfth" National Science and Technology Support Program (Grant No. 2014BAD04B03), Key Research and Development Special Foundation of Jiangsu Province (Grant No. BK2016309).

\section{References}

1 F. Ye, L. Zhang, X. Yin, Y. Zhang, L. Kong, Y. Liu and L. Cheng, J. Eur. Ceram. Soc., 2014, 34, 205-215. 
2 C. Montgomery, Technique of microwave measurements, McGraw-Hill Book Co, New York and London, 1947.

3 Y. Feng, T. Qiu and C. Shen, J. Magn. Magn. Mater., 2007, 318, 8-13.

4 T. Ting, R. Yu and Y. Jau, Mater. Chem. Phys., 2011, 126, 364368.

5 J. Hao, D. Kitts and S. b. Pandalai, Recent Res. Dev. Mol. Cell. Biochem., 2003, 1, 77-95.

6 P. Coronel, J. Simunovic, K. Sandeep, G. Cartwright and P. Kumar, J. Food Eng., 2008, 85, 528-536.

7 S. Dev, G. Raghavana and Y. Gariepya, J. Food Eng., 2008, 86, 207-214.

8 F. Tanaka, K. Morita, P. Mallikarjunan, Y. Hung and G. Ezeike, Trans. ASABE, 2002, 45, 733-740.

9 F. Tanaka, K. Morita, P. Mallikarjunan, Y. Hung and G. Ezeike, J. Food Eng., 2005, 71, 92-97.

10 X. Liao, G. Raghavan, J. Dai and V. Yaylayan, Food Res. Int., 2003, 36, 485.

11 X. Liao, G. Raghavan, G. Wu and V. Yaylayan, J. Mol. Liq., 2003, 107, 15-19.

12 N. Miura, S. Yagihara and S. Mashimo, J. Food Sci., 2003, 68, 1396-1403.
13 T. Motwani, K. Seetharaman and R. Anantheswaran, Carbohydr. Polym., 2007, 67, 73-79.

14 D. Fan, H. Shen, L. Huang, Y. Gao, H. Lian, J. Zhao, H. Zhang and W. Chen, Polymers, 2015, 7, 1895-1904.

15 D. Fan, L. Lin, L. Wang, L. Huang, B. Hu, X. Gu, J. Zhao and H. Zhang, Int. J. Biol. Macromol., 2017, 94, 266-270.

16 B. Roebuck, S. Goldblith and W. Westphal, J. Food Sci., 1972, 37, 199-204.

17 I. Huynen, N. Quiévy, C. Bailly, P. Bollen, C. Detrembleur, S. Eggermont, I. Molenberg, J. Thomassin, L. Urbanczyk and T. Pardoen, Acta Mater., 2011, 59, 3255-3266.

18 P. Bollen, N. Quiévy, I. Huynen, C. Bailly, C. Detrembleur, J. Thomassin and T. Pardoen, Scr. Mater., 2013, 68, 50-54.

19 X. Zhu, W. Guo and Z. Liang, Food Bioprocess Technol., 2015, 8, 1485-1494.

20 X. Wang, X. Huang, Z. Chen, X. Liao, C. Liu and B. Shi, J. Mater. Chem. C, 2015, 3, 10146-10153.

21 K. Tshabalala, S. Cho, J. Park, S. Pitale, I. Nagpure, R. Kroon, H. Swart and O. Ntwaeaborwa, J. Alloys Compd., 2011, 509, 10115-10120. 